\title{
Clinical Efficacy and Safety Profile of Anterior Thalamic Stimulation for Intractable Epilepsy
}

\author{
${ }^{1}$ Department of Neurosurgery, Institute of Psychiatry and Neurology, \\ Warszawa, Poland \\ 2 2nd Department of Neurology, Institute of Psychiatry and \\ Neurology, Warszawa, Poland \\ ${ }^{3}$ Department of Neuroradiology, Institute of Psychiatry and \\ Neurology, Warszawa, Poland \\ ${ }^{4}$ Department of Clinical Cytology, Centrum Medyczne Ksztalcenia \\ Podyplomowego, Warszawa, Poland
}

Michał Sobstyl $^{1} \quad$ Angelika Stapińska-Syniec ${ }^{1}$ Szczepan Iwański ${ }^{2} \quad$ Marcin Rylski ${ }^{3,4}$

J Neurol Surg A Cent Eur Neurosurg 2021;82:568-580.

\author{
Address for correspondence Angelika Stapińska-Syniec, MD, \\ Department of Neurosurgery, Instytut Psychiatrii i Neurologii, \\ Sobieskiego 9, Warsaw 02-957, Poland \\ (e-mail: angelika.stapinska@gmail.com).
}

\begin{abstract}
Keywords

- intractable epilepsy

- anterior nucleus of the thalamus

- deep brain stimulation

- thalamic stimulation

Introduction Deep brain stimulation of the anterior nucleus of the thalamus (ANT DBS) is a neuromodulation therapy for patients with refractory partial seizures. The ANT is the structure of a limbic system with abundant neuronal connections to temporal and frontal brain regions that participate in seizure propagation circuitry.

State of the Art We have performed a literature search regarding the clinical efficacy of ANT DBS. We discuss the surgical technique of the implantation of DBS electrodes with special attention paid to the targeting methods of the ANT. Moreover, we present in detail the clinical efficacy of ANT DBS, with a special emphasis on the stimulation parameters, a stimulation mode, and polarity. We also report all adverse events and present the current limitations of ANT DBS.

Clinical Implications In general, the safety profile of DBS in intractable epilepsy patients is good, with a low rate of surgery, hardware-related, and stimulation-induced adverse events. No significant cognitive declines or worsening of depressive symptoms was noted. At long-term follow-up, the quality-of-life scores have improved. The limitations of ANT DBS studies include a limited number of patients treated and mostly open-label designs with only one double-blind, randomized multicenter trial. Most studies do not report the etiology of intractable epilepsy or they include nonhomogeneous groups of patients affected by intractable epilepsy. There are no guidelines for setting initial stimulation parameters. All the variables mentioned may have a profound impact on the final outcome.

Conclusions ANT DBS appears to be a safe and efficacious treatment, particularly in patients with refractory partial seizures (three-quarters of patients gained at least $50 \%$ seizure reduction after 5 years). ANT DBS reduces most effectively the seizures originating in the temporal and frontal lobes. The published results of ANT DBS highlight promise and hope for patients with intractable epilepsy.
\end{abstract}

received

July 2, 2020

accepted after revision

December 16, 2020

published online

June 14, 2021 (c) 2021. Thieme. All rights reserved.

Georg Thieme Verlag KG,

Rüdigerstraße 14,

70469 Stuttgart, Germany
DOI https://doi.org/

10.1055/s-0041-1725954. ISSN 2193-6315. 


\section{Introduction}

Epilepsy affects $1 \%$ of the world population and despite the availability of antiepileptic pharmacologic treatment, drugresistant epilepsy remains poorly controlled in approximately one-third of patients. ${ }^{1,2}$ Most patients with refractory partial seizures benefit from resective surgeries, which may provide up to 80 to $90 \%$ reduction in seizure frequencies. ${ }^{3}$ However, up to $50 \%$ of patients suffering from intractable partial seizures are deemed not suitable candidates for resective surgeries. ${ }^{4}$ For this group of patients, neuromodulation therapies may constitute the last resort in the treatment of intractable epilepsy. Vagal nerve stimulation (VNS) has proven its efficacy as adjuvant therapy for intractable partial seizures. ${ }^{5}$ Given the high success rate of deep brain stimulation (DBS) in the past 25 years in the treatment of movement disorders, implementation of direct electrical brain stimulation to treat patients with intractable epilepsy began in research based on animal models of epilepsy. ${ }^{6-9}$

Different brain structures have been approached using DBS. $^{10}$ The anterior nucleus of the thalamus (ANT) gained widespread acceptance as a stereotactic target in the treatment of intractable epilepsy. ${ }^{8,9}$ The ANT is recognized as a key structure of the limbic system with abundant neuronal connections to temporal and frontal brain areas involved in seizure propagation circuitry. ${ }^{11}$ The best candidates for ANT DBS are patients with temporal epilepsy syndromes, not suitable for resective epilepsy surgery. ${ }^{8,9}$ Open-label studies and the largest double-blind randomized Stimulation of the Anterior Nucleus of the Thalamus for Epilepsy (SANTE) trial to date have shown a favorable mean seizure reduction in severely affected patients by intractable epilepsy. ${ }^{8,9,12-20}$ The ANT is the most often stimulated thalamic nucleus for the treatment of intractable epilepsy. $^{21-23}$ The second most common thalamic nucleus targeted for intractable epilepsy is the centromedian thalamic nucleus (CMN) ${ }^{10,22} \mathrm{CMN}$ represents a thalamic relay structure of the reticulocortical system that participates in wakefulness, affective processes, and widespread regulation of cortical excitability. ${ }^{10}$ It has been shown that CMN DBS is an effective treatment for tonic-clonic generalized seizures and atypical absences. Good results of CMN DBS have been obtained for Lennox-Gastaut syndrome. ${ }^{10,22}$

The origin of epileptogenic focus plays a role in selecting the stereotactic targets (ANT, CMN) for DBS procedures. The patients with epileptic discharges in the form of intractable partial seizures localized in the mesial-limbic (temporal) structures are selected for ANT DBS ${ }^{8,9}$ and those presented as tonic-clonic generalized seizures with mostly frontal localization are treated by CMN stimulation. ${ }^{10,22}$

The purpose of the review is to present the up-to-date knowledge regarding ANT DBS. We present the generally accepted indications and contraindications for ANT DBS as well the nuances of ANT targeting techniques including stereotactic trajectories (transventricular/extraventricular) to the ANT, imaging as well as postoperative documentation of implanted DBS leads before setting the stimulation parameters. We provide in detail the evidence of clinical efficacy, safety, and programming strategies regarding setting the initial stimulation parameters, stimulation polarity, as well as a stimulation mode. All surgery-, hardware-, and stimulation-related adverse events have been summarized. Neuropsychologic sequelae and limitations of ANT DBS are also discussed.

\section{Indications and Contraindications for ANT DBS}

It is still a challenging issue to identify the best surgical candidates for ANT DBS. Qualification for ANT DBS procedure involves a time-consuming presurgical multidisciplinary evaluation that includes video electroencephalographic (EEG) monitoring of habitual seizures, brain magnetic resonance imaging (MRI), detailed neuropsychologic assessment, as well as final interdisciplinary individual case description. Taking into consideration the pivotal role of ANT in the circuit of Papez, seizures originating from the mesial-limbic structures in patients who are not candidates for standard resective procedures seem to be the best candidates for ANT DBS. Patients with intractable epilepsy in whom a VNS or prior resective epilepsy surgery had failed (SANTE trial) have shown seizure reductions comparable to those individuals without these prior therapies. ${ }^{16}$ Salanova et al confirmed the safety and efficacy of ANT DBS in the 5-year follow-up study of patients with a previous VNS or resective epilepsy surgery. ${ }^{18}$ These clinical observations indicate that patients who had undergone VNS and resective epilepsy surgery remain good candidates for ANT DBS. Other predictors for the efficacy of ANT DBS are age at seizure onset, normal MRI without structural abnormalities, lateralized EEG abnormality, and positive performance in executive functions. ${ }^{8,18-20}$

The main contraindication for ANT DBS is the presence of a progressive neurologic etiology usually defined as a brain tumor, Rasmussen's encephalitis, or dementia. Other contraindications include a history of psychogenic seizures, depression or memory deficit, suicidal attempts, and psychosis unrelated to epilepsy. $9,12,15,16,18,20$ Cognitively impaired patients who are unable to complete a neuropsychologic assessment with an IQ $<70$ are excluded from ANT DBS. ${ }^{16,18}$ Contraindications for ANT DBS also include unknown seizure origin, unreliable seizure diary, inability to undergo strictly scheduled follow-up visits for optimization of ANT DBS therapy, and general MRI contraindications.

\section{Challenging Issues, due to Targeting and Trajectory Planning during ANT DBS}

One of the predictors for successful ANT DBS is the accurate placement of the DBS electrode, which ensures obtaining optimal therapeutic efficacy. ${ }^{24,25}$ In contrast to the functional procedures for movement disorders, an indirect targeting method (in reference to the anterior commissure/posterior commissure line) for stereotactic determination of the ANT may not be applicable in epilepsy surgery. ${ }^{24,25}$ It has been shown that patients with long-standing intractable epilepsy have disturbed brain architecture not only in the epileptic focus (foci) but also in the distinct brain areas, including the thalamic 
nuclei. The studies have revealed that the ANT atrophy is recognizable in patients with long-standing mesial temporal lobe epilepsy. ${ }^{26}$ Most authors point out that indirect stereotactic coordinates should be used cautiously and corrections should be made following the individual patient's anatomy. ${ }^{25,27}$ The application of the superimposition of the Wahren-Schaltenbrand atlas during targeting the ANT may also be difficult with quite variable final electrode placement confirmed by postoperative MRI. ${ }^{14}$ Direct visualization of the ANT borders and surrounding white matter tracts such as mammillothalamic tract (MTT), external and internal medullary lamina using short tau inversion recovery (STIR), or T1-weighted magnetization prepared gradient echo (MPRAGE) images may be difficult and requires considerable skills. $^{28}$ Fast gray matter acquisition $\mathrm{T} 1$ inversion recovery (FGATIR) is another 3-T MRI sequence that provides thin, high-resolution images with significantly better visualization of DBS targets when compared with standard 3-T T1- and T2-weighted images. ${ }^{29}$

The exact location of the best target within the ANT remains a matter of controversy. Some previous studies have suggested that a basolateral part of the ANT that corresponds to the anteroventral subdivision of the ANT is the most efficacious site of stimulation. ${ }^{19}$ Other authors have found that the optimal target point within the ANT lies more anterior, superior, and lateral to standard indirect targeting in relation to the midcommissural point, and refers to the anterior aspects of the ANT with broad connections to the frontal cortex, anterior cingulum, retrosplenial cortex, amygdala, and hippocampus. ${ }^{24,25}$ The search for the optimal ANT target requires further detailed studies that will correlate the exact anatomical location of active contact(s) with clinical outcome (-Fig. 1). In parallel to STN stimulation and posterior subthalamic area (PSA) stimulation, the stimulation of the top of MTT might be more efficacious than the ANT stimulation itself, as it targets thalamocortical radiation. ${ }^{30}$ In the study by Khan et al, two patients with gelastic seizures, due to hypothalamic hamartomas, achieved good seizure control under MTT stimulation. ${ }^{31}$ Another mechanism of DBS may be related to seizure control by the interruption of epileptogenic propagation in the MTT. ${ }^{30}$

In epilepsy surgery, it is not only the ANT targeting that is challenging but also the planning of stereotactic trajectories (-Fig. 2). Most authors used a transventricular rather than an extraventricular approach. ${ }^{25}$ Some authors stated that while using the extraventricular approach, implanting a DBS lead into the ANT is more difficult. ${ }^{25}$ Most authors used a transventricular approach by placing the burr holes just anterior or at coronal suture that enables that trajectories will most probably transverse ANT with a relatively high success rate. ${ }^{9,12,15,16,19,25}$ An alternative novel extraventricular approach with entry points (burr holes) placed in parietal regions has been recently proposed. ${ }^{32}$ With this approach, electrodes were placed bilaterally in the desired location in $90 \%$ of cases. Two or more contacts within the ANT were presented in $75 \%$ of all leads. ${ }^{32}$ In the recently published Medtronic Registry for Epilepsy (MORE) study, placing DBS leads correctly within the ANT was strictly associated

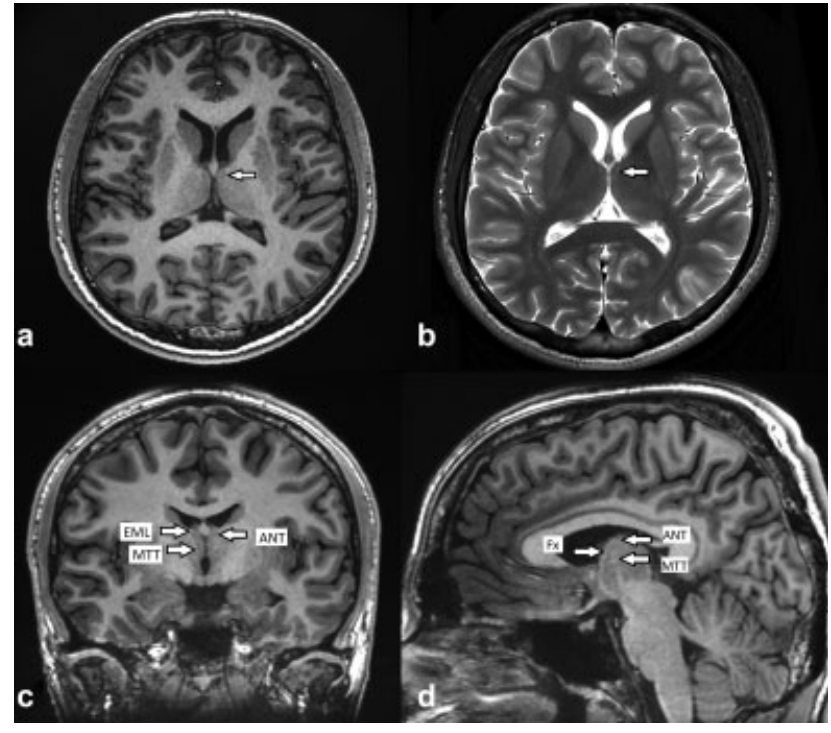

Fig. 1 The visualization of the anterior nucleus of the thalamus (ANT) in preoperative 3-T magnetic resonance imaging (MRI). (a) ANT is well demarcated in axial orientation in the T1-weighted sequence image (white arrow indicates the location of the left ANT). (b) The white arrow indicates the location of the left ANT in the T2-weighted sequence. (c) The coronal orientation showing the asymmetry of the ANT location on both sides on the T1-weighted image. The left ANT is larger than the right one and located more superiorly when compared with the right side. The white arrows on the right indicate the external medullary lamina (EML), and the mammillothalamic tract (MTT). (d) The parasagittal orientation depicting the same structures, the ANT and MTT as well as the fornix (Fx) on T1-weighted sequence.

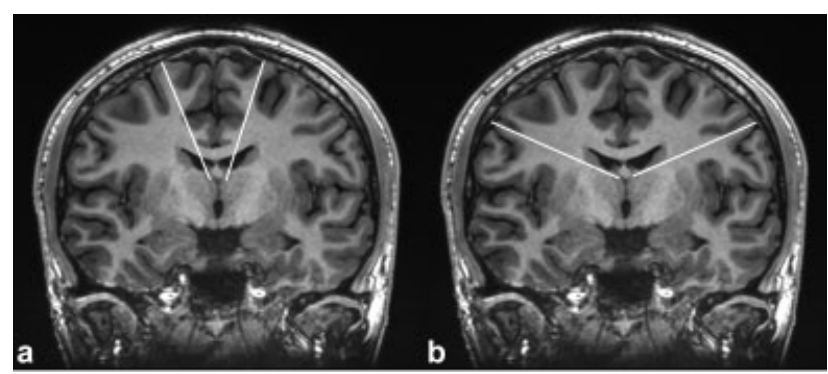

Fig. 2 (a) The transventricular trajectory to the anterior nucleus of the thalamus (ANT) transverses the gyrus frontalis superior, ventricle, and the dorsal thalamus. The entry points for the transventricular approach are placed at the front of the coronal suture. (b) The extraventricular trajectory to the ANT avoids the ventricular system transversion. This approach requires placing the stereotactic head frame low to plan the entry points ensuring that the extraventricular trajectory will not transverse the sulci. In some circumstances, planning the extraventricular trajectory may be challenging.

with a transventricular rather than an extraventricular trajectory. ${ }^{33}$ According to this study, $90 \%$ of transventricular lead trajectories had at least 1 contact in the ANT versus only $71 \%$ of extraventricular ones. The success rate of placing at least one contact in the ANT on both sides was $84 \%$ for transventricular trajectories and only 58\% for extraventricular trajectories. ${ }^{33}$ The results of this study support the selection of transventricular rather than extraventricular trajectories for placement of DBS leads in the ANT. 
Most patients operated on for epilepsy undergo DBS under local anesthesia with propofol sedation, although some patients are operated on under general anesthesia.9,12-20,28,31 The ANT may be identified by microrecording, but the microrecording signal is nonspecific. ${ }^{19}$ Microrecording may clearly show the entrance to the ANT if the transventricular approach is used. Another way of checking that all DBS lead contacts are within the thalamus is the measurement of individual contact impedances. The contacts located in the ventricle have low impedances when compared with the contacts that are within the brain tissue. To decrease the possibility of deviation of the DBS electrode during its introduction, a guiding cannula is usually inserted $10 \mathrm{~mm}$ above the calculated target. ${ }^{12,14,15,17,20}$ The introduction of a permanent DBS lead is done under fluoroscopic guidance. This ensures that the DBS lead is placed at the intended target. Perioperative 1.5-T MRI or computed tomography with the stereotactic frame on the patient's head fused with preoperative high-quality MRI enables localization of an implanted lead and excludes immediate intraoperative hemorrhagic complications. If no intraoperative imaging is performed, postoperative MR is mandatory. $9,12,15,16,24,25,27$

In contrast to functional procedures, a phenomenon of an insertional effect (microthalamotomy effect) is more often reported in patients who underwent ANT DBS. ${ }^{12,15,16,19}$ The microthalamotomy effect has been observed in patients with tremor-dominant movement disorders who received the DBS electrodes in the thalamus or the PSA. The incidence of the microthalamotomy effect in the reported studies after ANT DBS affects many patients and is more long lasting. In the SANTE trial, during a 3-month blinded phase, the insertional effect in a sham stimulation group decreased seizure frequency by $20 \%$ at 1 month and by $14 \%$ at 3 months. $^{16}$ The insertional effect may usually last from 2 to 4 months, followed by steady return of seizure frequency to baseline. ${ }^{19}$ However, a long-term insertional effect, lasting even 1 year after ANT DBS, was observed in individual patients. ${ }^{19}$ On the contrary, some authors did not observe this effect in their series. ${ }^{14}$ The targeting and surgical issues mentioned represent the current knowledge regarding ANT DBS surgery.

\section{Clinical Efficacy of ANT DBS}

Upton et al were the first authors to introduce ANT DBS for intractable epilepsy. ${ }^{8}$ In subsequent open-label studies, the seizure reduction ranged from 54 to $75.6 \%$ with a follow-up ranging from 10.6 to 43.8 months. ${ }^{9,12-14,19}$ In the uncontrolled study reported by Lee et al comprising six patients, subthalamic nucleus (STN) DBS in three patients resulted in $49.1 \%$ reduction of complex partial seizures and ANT DBS resulted in $75.4 \%$ reduction of seizures at a mean follow-up of 13.2 months. ${ }^{34}$ These results may indicate a higher reduction of a seizure rate in ANT DBS than in STN DBS. ${ }^{34}$ In the same year, Andrade et al reported a 60-month follow-up in six patients after ANT DBS who all were responders with at least 50\% complex partial seizure reduction. ${ }^{15}$ In the SANTE trial, by 2 years, there was a median $56 \%$ reduction in the seizure frequency, $54 \%$ of patients were responders with at least $50 \%$ seizure reduction. ${ }^{16}$ Five years after ANT DBS, the mean seizure reduction was $69 \%$, and the responder rate reached $68 \%$. In a 5 -year follow-up, $16 \%$ of patients were seizure free for at least 6 months. ${ }^{18}$ In the studies that followed the SANTE trial, the median seizure reduction ranged between 50 and $80.3 \%$, with a responder rate exceeding 70 to $80 \%$ of patients. ${ }^{17,19,20,28}$ The clinical efficacy of ANT DBS is presented in -Table 1.

\section{Other Factors (Seizure Origin, Previous VNS Therapy) Affecting the Outcome of ANT DBS}

Most studies on ANT DBS indicate that seizure outcome depends on the origin of the seizure. ${ }^{16,18-20}$ Seizures originating in one or both temporal lobes have a better prognosis than those originating in the frontal, parietal, or occipital lobes. ${ }^{21,28}$ The origin of the seizure focus may even have a stronger correlation with a long-term follow-up after ANT DBS. ${ }^{18}$ In the study by Salanova et al, after 5 years of ANT DBS, there was a $76 \%$ mean seizure reduction in the temporal origin seizures compared with a $59 \%$ mean seizure reduction in seizures originating from the frontal lobe. ${ }^{18}$ Patients with multiple epileptic foci responded less favorably than patients with temporal origin seizures. ${ }^{18}$ Other studies confirm that multifocal seizures with structural brain changes do not respond so favorably to ANT DBS. ${ }^{16,18-21,28}$

Apart from the seizure origin, a lot of variables may affect the outcome of ANT DBS. The subgroup of patients randomized in the SANTE trial had previous VNS therapy ( $44.5 \%$ of patients) or resective surgery for epilepsy (24.5\% of patients). ${ }^{18}$ These two variables did not affect the outcome regarding the mean seizure reduction when compared with patients without prior neuromodulation or resective surgery. The same conclusion may be drawn from the study by Kulju et al. ${ }^{35}$ In this study, the authors found that a progressive response to VNS predicts the response of ANT DBS. ${ }^{35}$ On the contrary, Park et al found favorable outcomes of ANT DBS in patients who had not responded to prior VNS. ${ }^{36}$ Although evidence for clinical effectiveness of VNS and DBS exists, the exact mechanisms of action remain unexplained. It seems that both neuromodulation therapies may affect thalamic activity indirectly (VNS) and directly (ANT DBS). ${ }^{35}$ Positron emission tomography (PET) studies during VNS have shown increased cerebral blood flow in the thalamus. $^{36}$ This increased synaptic thalamic activity may mediate the anticonvulsant effects of VNS therapy. ANT DBS directly modulates the thalamocortical activity and limbic seizure network. Further work is needed to elicit the effects of VNS and ANT DBS on epilepsy.

\section{Stimulation Parameters, Polarity, and Mode of ANT Stimulation}

The success of ANT DBS depends mainly on proper placement of DBS electrodes and programmed stimulation parameters. ${ }^{37}$ The stimulation parameters include voltage/amplitude, pulse frequency, duration, polarity, and stimulation mode (intermittent or continuous). There are no guidelines regarding the 


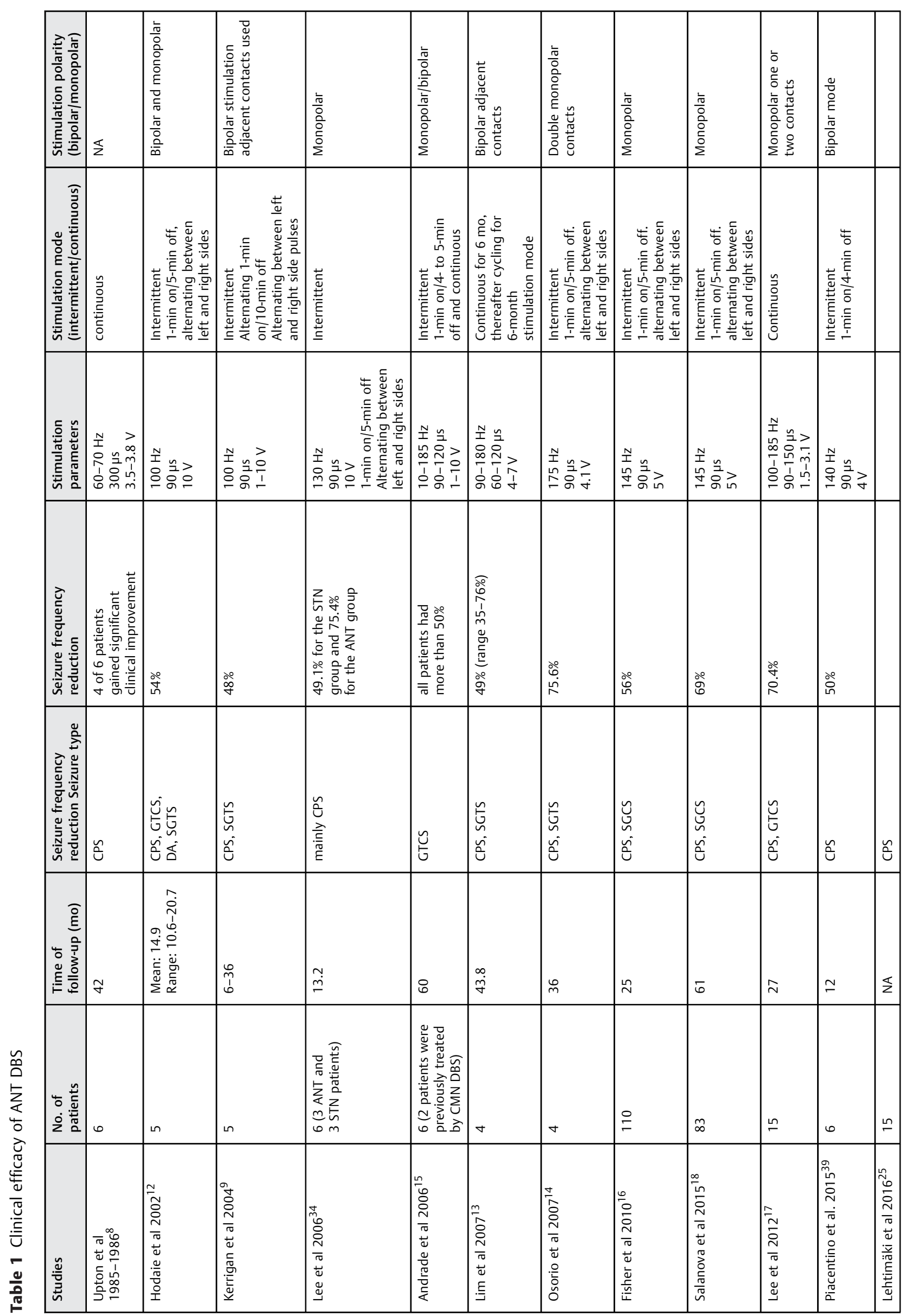




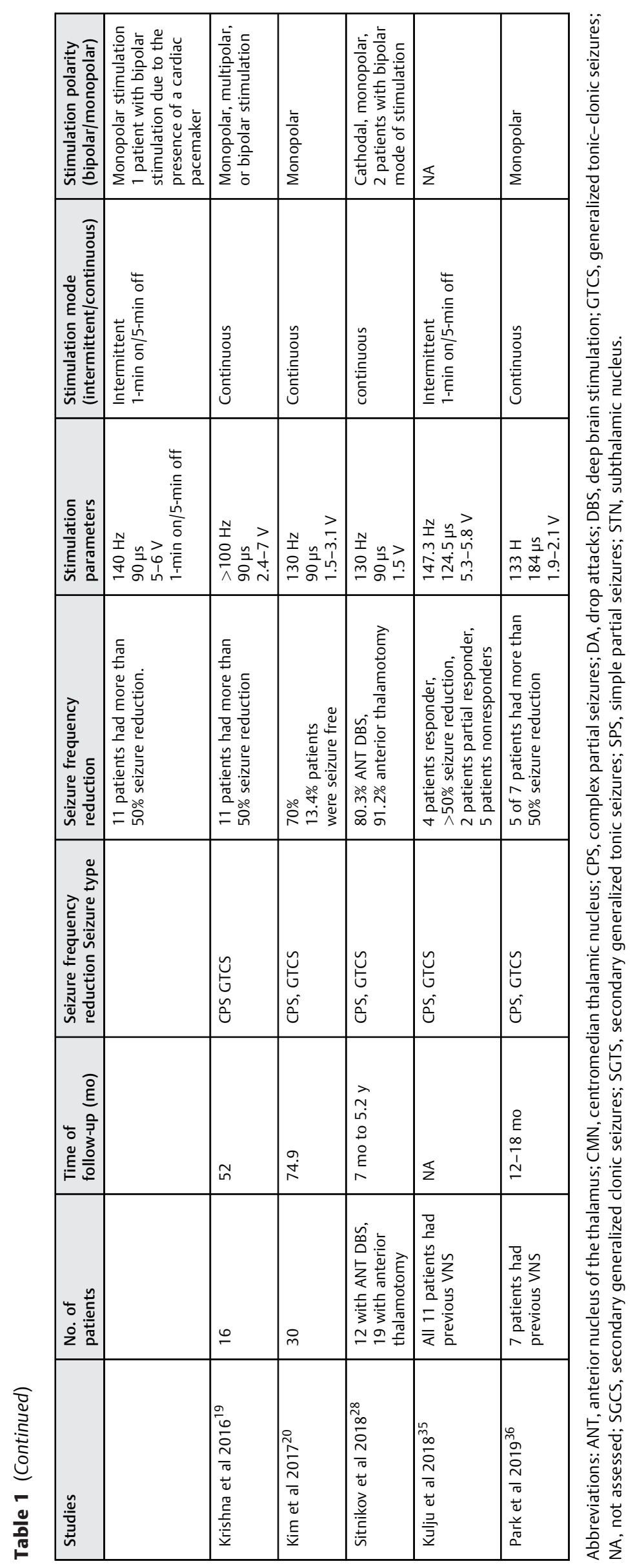


initial stimulation parameters in patients undergoing ANT DBS and various authors used different stimulation parameters, modes, and polarities ( - Table 1). The image-verified localization of implanted DBS leads may be of great importance for choosing appropriate contacts, stimulation polarity, and mode. ${ }^{16}$ It has been shown that improperly placed DBS leads have a suboptimal effect on seizure reduction and replacements were warranted. ${ }^{16}$ Moreover, stimulation of contacts outside the ANT may elicit unwanted psychiatric adverse events or cause subjective mood and memory problems. ${ }^{38}$

The antiepileptic effect of ANT DBS is based on animal studies and human data. ${ }^{6,7,12-20}$ In animal models, thalamic stimulation at low frequencies drives synchronization activity in distant brain regions, whereas stimulation at high frequencies desynchronizes intrinsic cortical activity. High-frequency thalamic stimulation can block epileptiform activity in the cortex. "Driving response" elicited by low-frequency ANT DBS is demonstrated on scalp EEG with synchronization of brain activity. Kerrigan et al found that eliciting driving response and determination of the threshold voltage for the driving response were good electrophysiologic markers of proper lead placement within the ANT. ${ }^{9}$ These authors concluded that the ability to evoke a driving response correlated with electrode placement in thalamic tissue, but not specifically in the ANT. The driving response may be elicited by the electrodes placed in other thalamic nuclei like the centromedian nucleus (CMN) or the dorsomedial nucleus (DMN). The neurophysiologic basis of a driving response and high-frequency related EEG desynchronization may guide in the choice of initial stimulation settings after ANT DBS. Hodaie et al found that the driving response is predictive of the successful outcome of ANT DBS therapy. ${ }^{12}$

Most studies including the SANTE trial used relatively high initial voltage $(\sim 5 \mathrm{~V})$. This may suggest that the initial stimulation parameters mentioned earlier could stimulate not only the ANT but also the surrounding brain tissue. Moreover, in most studies, the intermittent stimulation mode consisting of 1-minute-on stimulation followed by 5-minutes-off stimulation was used. ${ }^{9,14,16,18}$

As mentioned earlier, high-frequency stimulation above 100 to $130 \mathrm{~Hz}$ is used to desynchronize the EEG activity. The frequencies as high as $185 \mathrm{~Hz}$ have also been reported, but higher frequencies may induce unwanted adverse events and reduce the battery life significantly. ${ }^{37}$ The initial stimulation voltage is usually set in the range of 3 to $3.6 \mathrm{~V} .{ }^{37}$ Increasing the voltage above $3.6 \mathrm{~V}$ depletes the implantable pulse generator (IPG) more rapidly. To maintain a low effective voltage, the pulse width may be prolonged. Changing polarity from bipolar to one-contact monopolar or two-contact monopolar significantly lowers the stimulation voltage. Some authors have achieved mean seizure reduction ranging from 70 to $80.3 \%$ using a relatively low voltage of $1.5 \mathrm{~V}$ to as high as $3.1 \mathrm{~V}$ with cathodal monopolar stimulation. ${ }^{17,20,28}$ Low-voltage, monopolar stimulation mode appears more beneficial than high-voltage bipolar stimulation mode with mean seizure frequency reduction ranging from 46 to $56 \%{ }^{17,28}$ The pulse width in most studies is set above 90 microseconds. ${ }^{16,18,28}$

A longer pulse width requires a reduction of stimulation voltage to prevent the overstimulation of the ANT. It should also be noted that longer pulse widths are applied to deplete IPGs more rapidly. It is also speculated that longer pulse widths may stimulate the soma, whereas shorter pulse widths stimulate preferentially the axons. ${ }^{37}$

Polarity exerts a crucial impact on brain tissue activation around an implanted DBS lead. Most authors use monopolar or a mixture of monopolar and bipolar stimulation in individual patients. ${ }^{12,14,16,17}$ use of bipolar stimulation in ANT DBS is only rare reported. ${ }^{9,13,39}$ The advantage of bipolar stimulation is the more precise and consolidated volume of the stimulated brain tissue. ${ }^{37}$ The mode of stimulation is important for attenuating high cortical excitability that exists in patients with intractable epilepsy. Most studies use an intermittent mode of stimulation, but only a few implement a continuous stimulation mode. ${ }^{9,14,16,18,33,35}$ Clinical studies, where continuous stimulation mode has been used, report higher scores for mean seizure frequency reduction. $8,19,20,28$

\section{Adverse Events Related to ANT DBS Procedures}

Adverse events related to ANT DBS procedure can be divided into three categories: primarily operation-related complications (bleeding, venous infarction, improper intraoperative placement of DBS lead/s); hardware-related complications (infection, erosions, fracture, or migration of a DBS lead); and stimulation-induced complications (aggravation of seizure frequency or inducing de novo psychiatric/behavioral symptoms or aggravation of preexisting comorbid psychiatric/ behavioral symptoms). A low rate of adverse events of ANT DBS is explained by the fact that ANT DBS procedures have been performed by experienced functional stereotactic teams worldwide with extensive DBS expertise in movement disorders as well as in neuropsychiatric conditions. Adverse events related to ANT DBS are presented in - Table 2.

\section{Operation-associated Adverse Events in ANT DBS Procedures}

Hemorrhagic complications have been reported in patients treated by ANT DBS (- Table 2). ${ }^{13,16,20}$ In the study by Lim et al, of 4 patients, one patient (25\%) who received bilateral ANT and STN leads 2 days after the removal of STN leads experienced left-sided mild hand weakness caused by hemorrhage. ${ }^{13}$ In the SANTE trial, the authors reported five asymptomatic hemorrhages, representing $4.5 \%$ of all operated patients. ${ }^{16}$ In a study by Kim et al, one patient (3.4\%) experienced immediate left-sided hemiparesis caused by DBS lead insertion on the right side. $^{20}$ This weakness completely disappeared after 3 months with intensive physiotherapy. ${ }^{20}$ In the report of 31 patients ( 12 patients after ATN DNS and 19 after bilateral anterior thalamotomy) presented by Sitnikov et al, hemorrhages occurred in 2 patients (6.4\%) in each group. ${ }^{28}$ No reports of any patients suffering permanent neurologic deficit or death related to hemorrhage after ANT DBS can be found in the literature. ${ }^{16,21}$ Another operation-associated adverse event is the misplacement of DBS lead(s). ${ }^{9}$ In the SANTE trial, DBS leads outside the ANT were found in $8.2 \%$ of patients and subsequently replaced. ${ }^{16}$ A lower incidence of misplaced leads 


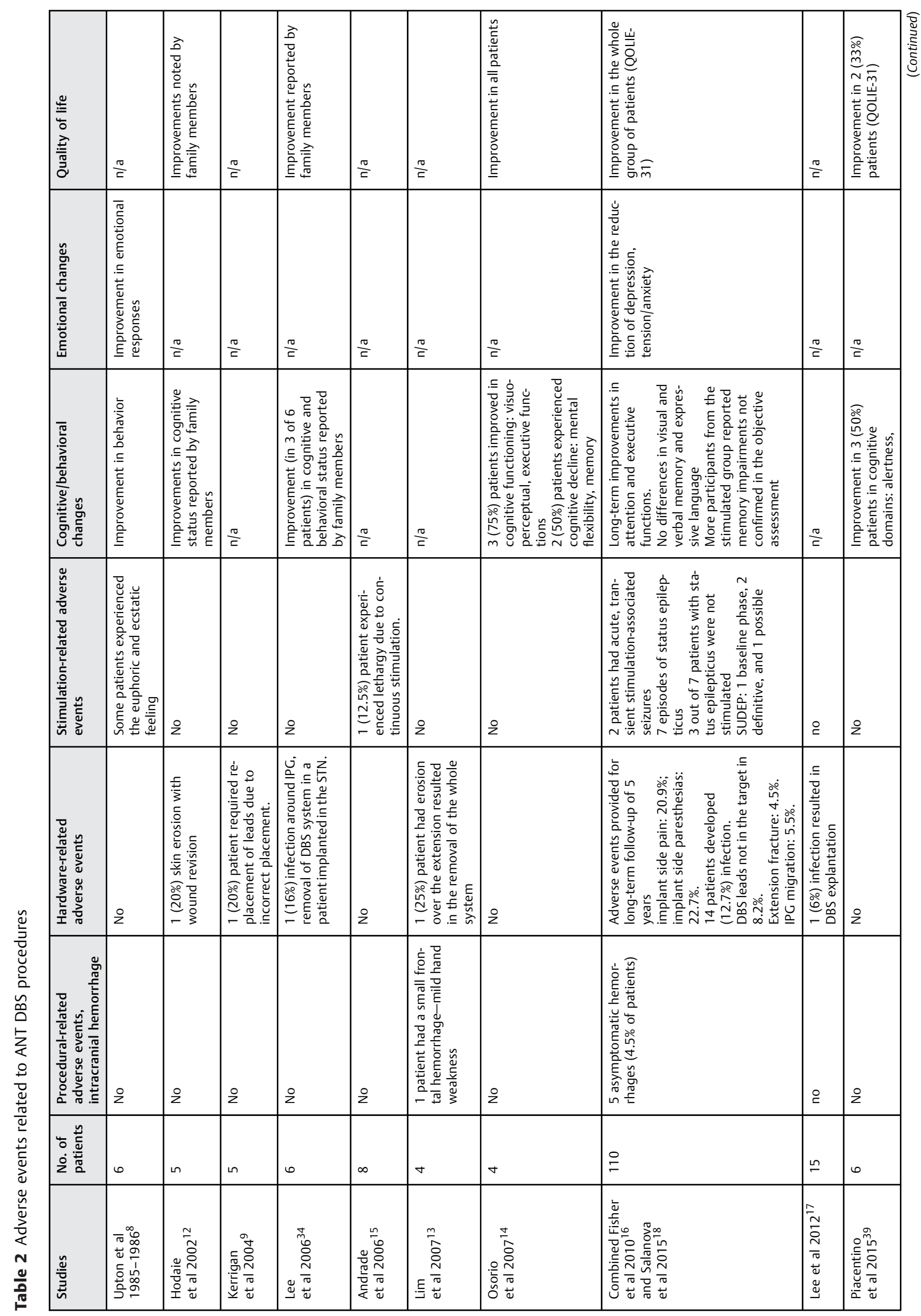




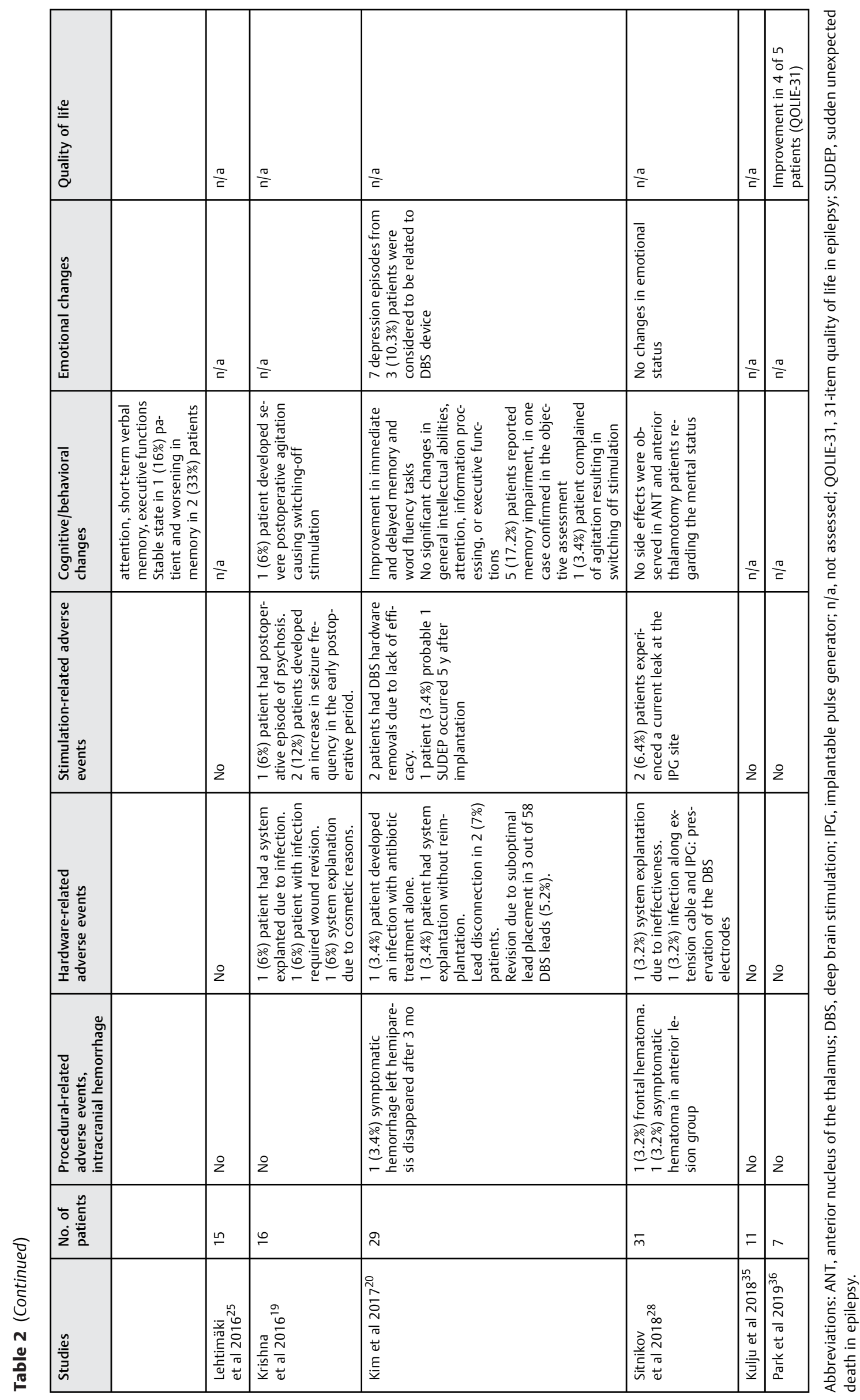


(3 of 58 DBS leads, 5.2\%) was observed in the study by Kim et $\mathrm{al}^{20}$ (- Table 2).

\section{Hardware-related Adverse Events Related to ANT DBS Procedures}

Skin complications related to the implanted DBS hardware were more common than hemorrhagic complications. Hodaie et al reported skin erosion that required wound revision without a further sequel in one patient $(25 \%){ }^{12}$ Lim et al removed the whole DBS system due to erosion located over the extension. ${ }^{13}$ In contrast to these two erosion episodes, infections were more common and difficult to treat. In the study by Lee et al, one patient (16\%) with STN DBS had one infection around the IPG, which resulted in the removal of the DBS system. ${ }^{17}$ In the study by Salanova et al, implant site infection was recognized in 14 patients (12.7\%). ${ }^{18}$ Krishna et al analyzed 16 patients with ANT DBS with a mean follow-up period of 52 months and found 2 infection episodes that required a full system removal in 1 patient (6\%). Other patients with a superficial infection had a wound revision with a further uneventful outcome. ${ }^{19}$

Apart from the infections that required removal of implanted DBS hardware, some patients requested removals due to the ineffectiveness of ANT DBS in reducing seizure frequency or for cosmetic reasons. In the study by Krishna et al, one patient (6\%) requested DBS hardware removal for cosmetic reasons. ${ }^{19}$ In the study by Kim et al, 7 of 29 patients (24\%) were nonresponders and DBS hardware was removed due to lack of efficacy in 2 patients $(6.8 \%) .{ }^{20}$ In the study by Sitnikov et al, 1 of 12 patients (8.3\%) did not respond to ANT DBS and subsequently underwent DBS system explantation. ${ }^{28}$ Another adverse event related to the implanted DBS hardware was excessive pain and unpleasant sensory changes at the implant side. ${ }^{18,20}$ Sitnikov et al observed that 2 of 12 (16.6\%) patients experienced a current leak at the IPG site that resolved after changing the stimulation polarity from a monopolar to a bipolar configuration. ${ }^{28} \mathrm{~A}$ monopolar stimulation may increase unpleasant long-lasting sensory disturbances at the IPG site. ${ }^{16,18,28}$ Other authors who used a bipolar configuration did not report this type of complication..$^{9,13,39}$ Other less common hardware-related problems were the fractures of DBS system elements. Kim et al found DBS lead fractures in 2 of 29 patients $(6.9 \%) .{ }^{20}$ Salanova et al reported that the fractures of extensions were found in $4.5 \%$ of 83 patients. $^{18}$

\section{Stimulation-induced Adverse Events Related to ANT DBS}

ANT DBS aims at reducing seizure frequency in patients with intractable epilepsy. Some studies provide evidence that stimulation in individual patients may provoke frequent seizures. In the SANTE trial, Fisher et al found acute, stimulus-linked seizures in two patients (1.8\%). ${ }^{16}$ Lowering stimulation voltage reduced seizures in these two patients. Fisher et al emphasized that close monitoring of patients after the initiation of ANT stimulation should be advocated. ${ }^{16}$ Interestingly, no other patients showed "kindling" with delayed emergence of seizures related to the stimulation increase over time during the followup period. Also, Krishna et al reported that two patients (12.5\%) had an increased seizure frequency in an early postoperative period. $^{19}$

\section{Cognitive and Emotional Sequelae of ANT DBS}

The ANT is a relay structure of the circuit of Papez. ${ }^{40}$ stimulation-related adverse events may be elicited by stimulation of the limbic system and affect memory capacities as well as behavioral changes. There is a possibility of de novo induction or aggravation of preexisting psychiatric symptoms. ${ }^{25,35,36,38}$ A group of patients with long-standing intractable epilepsy is handicapped by memory and psychiatric side effects related to uncontrolled seizures. Psychiatric and neuropsychologic assessments play a major role in selecting and following patients after DBS for intractable epilepsy. ${ }^{16,18,21}$ The first report by Osorio et al highlighted a decline in memory and mental flexibility at 12 months of follow-up. ${ }^{14}$ On the contrary, in a group of 9 patients followed with a mean of 15.9 months after ANT DBS, Oh et al found favorable results for delayed memory and verbal fluency with no effect on information processing, executive function, or general cognitive functioning. ${ }^{41}$ They stated that the improvements in verbal recall and verbal fluency may be related to activation of the frontolimbic circuit. ${ }^{41}$

The SANTE trial has revealed gradual improvement in the cognitive domains of attention and executive functions. ${ }^{18}$ Kim et al found verbal memory and word fluency improvements in 12 of 29 patients at 12 months of neuropsychological follow-up. ${ }^{20}$ Tröster et al, following patients for 7 years after ANT DBS from the previously reported prospective randomized SANTE trial, found no significant cognitive declines or worsening of depression. ${ }^{17}$ Järvenpää et al found the reversibility of psychiatric adverse events in 4 of 22 patients followed for at least 1 year after ANT DBS. Changing the stimulation parameters or active stimulating contacts resolved these psychiatric adverse events. ${ }^{38}$ These authors highlight the importance of proper selection of stimulating contacts within ANT because patients troubled by psychiatric adverse events had DBS leads implanted mostly beneath the ANT.

In contrast to the above-mentioned studies, Novais et al found that the predisposing factors for developing de novo psychopathology constitute generalized epileptogenic activity and the ANT DBS procedure itself. ${ }^{42}$ ANT DBS is considered to be adaptable and reversible. One report describing a patient with persistent psychiatric adverse events contradicts this belief. $^{43}$

Mood disturbances or memory problems observed only in a blinded phase of the SANTE trial may be related to overstimulation of the ANT and a lesional (microthalamotomy) effect. ${ }^{16}$ Monopolar stimulation using relatively high stimulation parameters (especially voltage) in an early postoperative phase may explain this transient subjective mood and memory problems. ${ }^{16}$ Nevertheless, the long-term neuropsychologic outcomes have shown improvements in memory, verbal fluency, and mood in patients after ANT DBS. ${ }^{44}$ Other authors who used ANT DBS noticed objective improvements of 
alertness and communicative behavior. ${ }^{36,41,44}$ ANT DBS improves some cognitive domains without major mood and memory disorders, whereas bilateral anterior thalamotomy has no significant changes in mental and emotional states in the group of 19 patients in the report by Sitnikov et al. ${ }^{28}$

\section{Limitations of ANT DBS for Intractable Epilepsy}

Although the ATN is the most frequently targeted thalamic nucleus for intractable epilepsy, fewer than 250 patients have been treated by bilateral ANT DBS so far. ${ }^{8,9,12-20}$ Nevertheless, three-quarters of the patients observed for more than 5 years gained at least $>50 \%$ seizure reduction. ${ }^{17-20}$ Apart from a still limited number of patients treated, there are other limitations related to ANT DBS. There is only one double-blind, multicenter, randomized trial (SANTE trial). other reports of the ANT are open-label studies, usually single center and retrospective. ${ }^{16-20}$ A limited number of treated patients and study designs preclude a meta-analysis of the data regarding ANT DBS for intractable epilepsy.

Another drawback of ANT DBS is lack of guidelines regarding the initial stimulation parameters. ${ }^{8-20}$ Various multidisciplinary DBS teams use different stimulation parameters (voltages, frequencies, and pulse widths), as well as stimulation polarities and a stimulation mode (intermittent or continuous). Setting various stimulation parameters is a confounding factor and may exert a profound impact on the final outcome. The reported patient groups are not homogenous with regard to etiology and clinical presentations of epilepsy. $8,15,19,20,24,28$ Some reports include patients with unifocal or multifocal MRI abnormalities and different origins of epilepsy (temporal, frontal, parietal). Despite all these variables, placing a DBS lead correctly plays a major role in achieving a good clinical outcome. $^{16,32,33}$ The issue of surgical approaches used in ANT DBS is highlighted in the MORE study, showing that a transventricular rather than an extraventricular approach is more efficacious in placing DBS leads correctly at the ANT. ${ }^{33}$

The ANT is a relatively small structure located at the dorsal surface of the thalamus with visible differences in shape and location between the right and left cerebral hemispheres in individual patients. The differences in the ANT volumes may be attributable to long-standing intractable epilepsy with secondary atrophy of this relay nucleus in the circuit of Papez. ${ }^{11,26,40}$ Modern MRI sequences may greatly facilitate direct targeting of the ANT. ${ }^{24,27}$ Software that enables precise visualization of the implanted DBS leads may guide and optimize the selection of active contact(s) for the initial stimulation. These achievements may have a positive influence on seizure reduction.

Another important limitation of ANT DBS is the possibility of the occurrence of life-threatening complications due to longstanding intractable seizures even after ANT DBS procedures. These complications include the Sudden Unexpected Death in Epilepsy (SUDEP) and status epilepticus (SE).SUDEP and SE may be controlled only after resective epilepsy surgery with total seizure freedom. It means that ANT DBS is still not a curative but a palliative treatment modality for patients affected by severe intractable seizures. SUDEP is a fatal complication of epilepsy and is defined as the sudden and unexpected or nontraumatic, nondrowning death without a toxicological or anatomical cause of death detected during the postmortem examination. SUDEP is responsible for $50 \%$ of all deaths in refractory epilepsy. Proposed pathophysiologic mechanisms include seizureinduced cardiac and respiratory arrests.

Long-term clinical trials confirm the observation that life-threatening complications of long-standing intractable epilepsy (SUDEP and SE) are not completely eliminated. ${ }^{16,18,20}$ In the study by Salanova et al, seven deaths occurred over the follow-up period. None of them were related to the implanted device as assessed by the investigator or data monitoring committee. One death occurred in a baseline phase, whereas two definitive and one probable SUDEP occurred after ANT DBS. ${ }^{18}$ In this study, the rate of definitive/probable SUDEP of 2.9 per 1,000 patient-years was more favorable than the published rates as high as 9.3 per 1,000 patient-years in epilepsy surgical patients. ${ }^{18}$ In another study reported by Kim et al with a mean follow-up of 74.9 months, five deaths were noted. One (3.4\%) probable SUDEP occurred 5 years after the ANT DBS implantation. ${ }^{20}$

SE was also observed in patients after ANT DBS. In the SANTE trial, five (4.5\%) patients experienced SE. In the longterm continuation of the SANTE trial, Salanova et al reported additional two patients affected by SE. ${ }^{18}$ Six of seven patients required hospitalization, due to $S E$. Three of the seven events of SE occurred in patients who were not stimulated, which may suggest that discontinuation of ANT DBS may be a provoking factor. ${ }^{18}$ Four of seven SE events were nonconvulsive in nature. ${ }^{18}$

The last concern is related to suicidal ideation after ANT DBS observed in studies with sufficient long-term follow-up. ${ }^{16,18}$ Although the history of suicidal attempts or suicidal ideation with active depression constitutes the known contraindications for ANT DBS, these problems may occur following ANT DBS. In the study by Salanova et al, $8.2 \%$ of patients reported episodes of suicidal ideation after 5 years. One patient committed suicide 4 years after ANT DBS. ${ }^{18}$ Most patients (10 of 13) with suicidal ideation suffered from depression. The causes of suicidal ideation were multifactorial and were not related to DBS hardware or its malfunction. ${ }^{18}$ Lim et al also reported a patient who committed suicide after ANT DBS. ${ }^{13}$

Taking these clinical observations into consideration, patients and their caregivers should be informed about these possible life-threatening conditions (SUDEP, SE) and the risk of suicidal ideation episodes mostly related to depression after ANT DBS.

\section{Conclusion}

ANT DBS has shown its efficacy in treating patients with intractable epilepsy who failed other treatment modalities. ANT DBS remains a safe functional neurosurgical procedure with minimal or transient adverse events. Hemorrhagic complications were reported only in a few cases. There were no reported permanent neurologic deficits or deaths related to surgery. In a few patients, skin-related complications resulted in infections with subsequent hardware explantation. Social 
functioning and quality of life have improved after ANT DBS. The limitations of ANT DBS include a small number of patients treated and a lack of guidelines regarding the initial stimulation parameters as well as a stimulation mode. Although ANT DBS is an effective treatment, it does not preclude the occurrence of life-threatening complications related to longstanding intractable epilepsy. SUDEP and SE can be minimized only by complete seizure control. Postoperative episodes of suicidal ideation or attempted suicides raise some concerns. ANT DBS should be regarded as palliative treatment in patients severely affected by intractable epilepsy, but it is not curative. Optimization of targeting technique based on available highresolution MRI and precise localization of implanted electrodes may guide imaging-verified contact(s) selection for setting stimulation parameters and enhance ATN DBS efficacy, all of which may optimize ANT DBS therapy.

\section{Highlights}

- ANT DBS appears to be a safe and efficacious treatment, particularly in patients with refractory partial seizures.

- ANT DBS most effectively reduces the seizures originating in the temporal and frontal lobes.

- ATN DBS has a relatively safe neuropsychologic profile with increased quality-of-life improvement followed for years after thalamic stimulation.

- ANT DBS is a palliative, not curative, treatment, but it is effective in patients in whom VNS and resective epilepsy surgery failed to control intractable seizures.

\section{Conflict of Interest}

None declared.

\section{References}

1 Kwan P, Brodie MJ. Early identification of refractory epilepsy. N Engl J Med 2000;342(05):314-319

2 Brodie MJ, Dichter MA. Established antiepileptic drugs. Seizure 1997;6(03):159-174

3 Téllez-Zenteno JF, Dhar R, Wiebe S. Long-term seizure outcomes following epilepsy surgery: a systematic review and meta-analysis. Brain 2005;128(Pt 5):1188-1198

4 A global survey on epilepsy surgery, 1980-1990: a report by the Commission on Neurosurgery of Epilepsy, the International League Against Epilepsy. Epilepsia 1997;38(02):249-255

5 Scherrmann J, Hoppe C, Kral T, Schramm J, Elger CE. Vagus nerve stimulation: clinical experience in a large patient series. J Clin Neurophysiol 2001;18(05):408-414

6 Mirski MA, Rossell LA, Terry JB, Fisher RS. Anticonvulsant effect of anterior thalamic high frequency electrical stimulation in the rat. Epilepsy Res 1997;28(02):89-100

7 Mirski MA, Ferrendelli JA. Interruption of the connections of the mammillary bodies protects against generalized pentylenetetrazol seizures in guinea pigs. J Neurosci 1987;7(03):662-670

8 Upton AR, Cooper IS, Springman M, Amin I. Suppression of seizures and psychosis of limbic system origin by chronic stimulation of anterior nucleus of the thalamus. Int J Neurol1985-198619-20:223-230

9 Kerrigan JF, Litt B, Fisher RS, et al. Electrical stimulation of the anterior nucleus of the thalamus for the treatment of intractable epilepsy. Epilepsia 2004;45(04):346-354
10 Klinger NV, Mittal S. Clinical efficacy of deep brain stimulation for the treatment of medically refractory epilepsy. Clin Neurol Neurosurg 2016;140:11-25

11 Child ND, Benarroch EE. Anterior nucleus of the thalamus: functional organization and clinical implications. Neurology 2013;81(21):1869-1876

12 Hodaie M, Wennberg RA, Dostrovsky JO, Lozano AM. Chronic anterior thalamus stimulation for intractable epilepsy. Epilepsia 2002;43(06):603-608

13 Lim SN, Lee ST, Tsai YT, et al. Electrical stimulation of the anterior nucleus of the thalamus for intractable epilepsy: a long-term follow-up study. Epilepsia 2007;48(02):342-347

14 Osorio I, Overman J, Giftakis J, Wilkinson SB. High frequency thalamic stimulation for inoperable mesial temporal epilepsy. Epilepsia 2007;48(08):1561-1571

15 Andrade DM, Zumsteg D, Hamani C, et al. Long-term follow-up of patients with thalamic deep brain stimulation for epilepsy. Neurology 2006;66(10):1571-1573

16 Fisher R, Salanova V, Witt T, et al; SANTE Study Group. Electrical stimulation of the anterior nucleus of thalamus for treatment of refractory epilepsy. Epilepsia 2010;51(05):899-908

17 Lee KJ, Shon YM, Cho CB. Long-term outcome of anterior thalamic nucleus stimulation for intractable epilepsy. Stereotact Funct Neurosurg 2012;90(06):379-385

18 Salanova V, Witt T, Worth R, et al; SANTE Study Group. Long-term efficacy and safety of thalamic stimulation for drug-resistant partial epilepsy. Neurology 2015;84(10):1017-1025

19 Krishna V, King NK, Sammartino F, et al. Anterior nucleus deep brain stimulation for refractory epilepsy: insights into patterns of seizure control and efficacious target. Neurosurgery 2016;78 (06):802-811

$20 \mathrm{Kim}$ SH, Lim SC, Kim J, Son BC, Lee KJ, Shon YM. Long-term followup of anterior thalamic deep brain stimulation in epilepsy: a 11year, single center experience. Seizure 2017;52:154-161

21 Ooi YC, Styliaras JC, Sharan A. Thalamic stimulation for epilepsy. Neurosurg Clin N Am 2011;22(04):457-464, v-vi

22 Salanova V. Deep brain stimulation for epilepsy. Epilepsy Behav 2018;88S:21-24

23 Schuepbach WM, Rau J, Knudsen K, et al; EARLYSTIM Study Group. Neurostimulation for Parkinson's disease with early motor complications. N Engl J Med 2013;368(07):610-622

24 Möttönen T, Katisko J, Haapasalo J, et al. Defining the anterior nucleus of the thalamus (ANT) as a deep brain stimulation target in refractory epilepsy: delineation using 3 T MRI and intraoperative microelectrode recording. Neuroimage Clin 2015;7 (07):823-829

25 Lehtimäki K, Möttönen T, Järventausta K, et al. Outcome based definition of the anterior thalamic deep brain stimulation target in refractory epilepsy. Brain Stimul 2016;9(02):268-275

26 Keller SS, O'Muircheartaigh J, Traynor C, Towgood K, Barker GJ, Richardson MP. Thalamotemporal impairment in temporal lobe epilepsy: a combined MRI analysis of structure, integrity, and connectivity. Epilepsia 2014;55(02):306-315

27 Jiltsova E, MöttönenT, Fahlström M, et al. Imaging of anterior nucleus of thalamus using 1.5T MRI for deep brain stimulation targeting in refractory epilepsy. Neuromodulation 2016;19(08):812-817

28 Sitnikov AR, Grigoryan YA, Mishnyakova LP. Bilateral stereotactic lesions and chronic stimulation of the anterior thalamic nuclei for treatment of pharmacoresistant epilepsy. Surg Neurol Int 2018; 9:137

29 Sudhyadhom A, Haq IU, Foote KD, Okun MS, Bova FJ. A high resolution and high contrast MRI for differentiation of subcortical structures for DBS targeting: the fast gray matter acquisition $\mathrm{T} 1$ inversion recovery (FGATIR). Neuroimage 2009;47(Suppl 2):T44-T52

30 Zumsteg D, Lozano AM, Wennberg RA. Mesial temporal inhibition in a patient with deep brain stimulation of the anterior thalamus for epilepsy. Epilepsia 2006;47(11):1958-1962 
31 Khan S, Wright I, Javed S, et al. High frequency stimulation of the mamillothalamic tract for the treatment of resistant seizures associated with hypothalamic hamartoma. Epilepsia 2009;50 (06):1608-1611

32 Wang YC, Grewal SS, Middlebrooks EH, et al. Targeting analysis of a novel parietal approach for deep brain stimulation of the anterior nucleus of the thalamus for epilepsy. Epilepsy Res 2019;153:1-6

33 Lehtimäki K, Coenen VA, Gonçalves Ferreira A, et al; MORE investigators. The Surgical Approach to the Anterior Nucleus of Thalamus in Patients With Refractory Epilepsy: Experience from the International Multicenter Registry (MORE). Neurosurgery 2019;84(01):141-150

34 Lee KJ, Jang KS, Shon YM. Chronic deep brain stimulation of subthalamic and anterior thalamic nuclei for controlling refractory partial epilepsy. Acta Neurochir Suppl (Wien) 2006;99:87-91

35 Kulju T, Haapasalo J, Lehtimäki K, Rainesalo S, Peltola J. Similarities between the responses to ANT-DBS and prior VNS in refractory epilepsy. Brain Behav 2018;8(06):e00983

36 Park HR, Choi SJ, Joo EY, et al. The role of anterior thalamic deep brain stimulation as an alternative therapy in patients with previously failed vagus nerve stimulation for refractory epilepsy. Stereotact Funct Neurosurg 2019;97(03):176-182

37 Jaseja H. Deep brain stimulation in intractable epilepsy: postulated optimal stimulation parameters. Epilepsy Behav 2013;29 (03):597-598
38 Järvenpää S, Peltola J, Rainesalo S, Leinonen E, Lehtimäki K, Järventausta K. Reversible psychiatric adverse effects related to deep brain stimulation of the anterior thalamus in patients with refractory epilepsy. Epilepsy Behav 2018;88:373-379

39 Piacentino M, Durisotti C, Garofalo PG, et al. Anterior thalamic nucleus deep brain stimulation (DBS) for drug-resistant complex partial seizures (CPS) with or without generalization: long-term evaluation and predictive outcome. Acta Neurochir (Wien) 2015; 157(09):1525-1532, discussion 1532

40 Papez J. A proposed mechanism of emotion. Arch Neurol Psychiatry $1937 ; 38: 725-743$

41 Oh YS, Kim HJ, Lee KJ, Kim YI, Lim SC, Shon YM. Cognitive improvement after long-term electrical stimulation of bilateral anterior thalamic nucleus in refractory epilepsy patients. Seizure 2012;21(03):183-187

42 Novais F, Pestana LC, Loureiro S, Andrea M, Figueira ML, Pimentel J. Predicting de novo psychopathology after epilepsy surgery: a 3year cohort study. Epilepsy Behav 2019;90:204-208

43 Doležalová I, Kunst J, Kojan M, Chrastina J, Baláž M, Brázdil M. Anterior thalamic deep brain stimulation in epilepsy and persistent psychiatric side effects following discontinuation. Epilepsy Behav Rep 2019;12:100344

44 Tröster AI, Meador KJ, Irwin CP, Fisher RSSANTE Study Group. Memory and mood outcomes after anterior thalamic stimulation for refractory partial epilepsy. Seizure 2017;45:133-141 